\title{
Conductive Porous Polymer Synthesis via a Novel Atmospheric Pressure Plasma Polymerization and Its Application to Gas Sensors
}

\author{
Choon-Sang Park' ${ }^{1}$, Do Yeob Kim², Eun Young Jung ${ }^{1}$, Dong Ha Kim¹, Hyung-Kun Lee ${ }^{2}$, \\ Daseulbi Kim', Gyu Tae Bae', Jeong Hyun Seo ${ }^{3}$, Bhum Jae Shin', ${ }^{4}$ eung-Sik Tae ${ }^{1}$ \\ ${ }^{1}$ School of Electronics Engineering, College of IT Engineering, Kyungpook National University, Daegu \\ 41566, Republic of Korea \\ 2 ICT Materials and Components Research Laboratory, Electronics and Telecommunications \\ Research Institute, Daejeon 34129, Republic of Korea \\ ${ }^{3}$ Department of Electronics Engineering, Incheon National University, Incheon 22012, Republic of \\ Korea \\ ${ }^{4}$ Department of Electronics Engineering, Sejong University, Seoul 05006, Republic of Korea \\ purplepcs@ee.knu.ac.kr, hstae@ee.knu.ac.kr
}

\begin{abstract}
:
New nanostructured conductive porous polymers are prepared by novel atmospheric pressure plasma jets (APPJs) polymerization technique, and their $\mathrm{NO}_{2}$-sensing properties are investigated in this study. This plasma polymerization synthesis technique can grow the poly- and single-crystalline high-density nanoparticles with a fast deposition rate under room-temperature process by improving the sufficient nucleation and recombination of the various monomers, such as aniline and thiophene, using a novel APPJs technique. To check the suitability for sensing materials of gas sensors, the resistance variations of the plasma-polymerized aniline and thiophene nanoparticles grown on the interdigitated electrodes are examined by doping the iodine. As a result, the proposed APPJs device can produce the high density, porous, and ultra-fast polymer films, and polymers based gas sensors have high sensitivities at room temperature.
\end{abstract}

Key words: conductive polymer, porous structure, gas sensors, $\mathrm{NO}_{2}$, polyaniline, polythiophene

\section{Introduction}

Recently, conductive polymers, such as polyaniline (PANI), polythiophene (PTh), and their derivatives, have attracted attention to researchers as sensing materials of gas sensors [1]. In comparison with most of the commercial gas sensors, based usually on metal oxides and operated at high temperatures, the gas sensors made of conductive porous polymers have many advantages. The advantages of conductive polymers compared to inorganic materials used until now are their diversity, their easy synthesis and particularly, their sensitivity at room temperature [2]. Conductive polymers can be synthesized by various techniques such as chemical synthesis, electrochemical method, hard and soft templates, interfacial polymerization, and plasma polymerization [3]. However, almost all of polymerization techniques have high synthesis temperatures with solution or wet process. Therefore, conventional conductive polymers have generally amorphous characteristics with non-porous properties due to high temperature process, which would have difficulty realizing highly sensitive gas sensors. We have recently developed a nano-porous polymer synthesis method using a novel atmospheric pressure plasma jets (APPJs) with additional guide tube and bluff body systems [4]. The plasma polymerizations of aniline, pyrrole, and thiophene have been successfully implemented using intense and broad glow-like plasma [4, 5]. However, the interactions between the use of the conductive nano-porous polymers prepared by novel APPJs method and the gas-sensing characteristics at room temperature conditions are not well known. Accordingly, this study investigates the $\mathrm{NO}_{2}$ sensing properties of the iodine $\left(I_{2}\right)$ doped conductive porous PANI and PTh films grown on the interdigitated electrode in order to check 

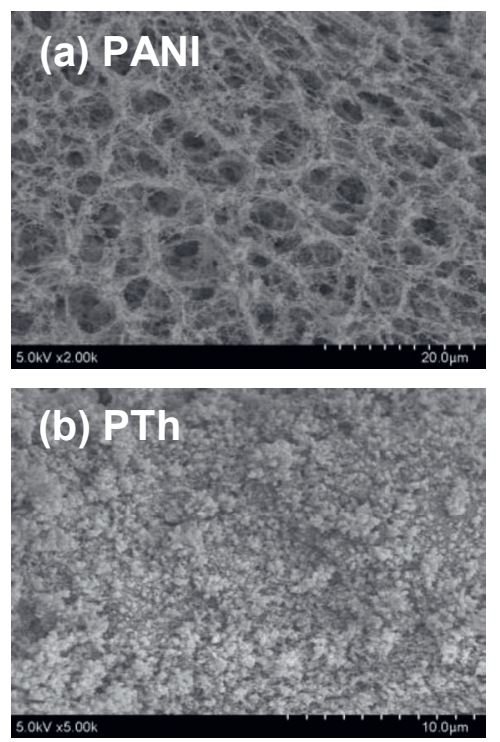

Fig. 1. SEM images of plasma-polymerized aniline (a) and thiophene (b) thin films prepared using novel $A P P J s$ after 10 min deposition.

the suitability for sensing materials of gas sensors.

\section{Experimental}

In order to produce intense glow-like plasma by using novel APPJs, we use a guide tube and substrate on polytetrafluoroethylene (PTFE) bluff body installed at the jet end to confine the jet flow and to minimize the quenching from ambient air. Consequently, the novel APPJs can expand and extend farther downstream in nucleation region and can produce the broaden and intense glow-like plasma during plasma polymerization. The plasma polymerization method using novel APPJs source with a guide tube and bluff body was previously described in detail [4-6]. Argon gas was employed as the discharge gas for plasma generation and its flow rate was in the range of $1700 \mathrm{sccm}$. Liquid aniline or thiophene monomer was vaporized by means of a glass bubbler which was supplied by argon gas with flow rates in the range of 160 $\mathrm{sccm}$. The nano size polymer was obtained at a sinusoidal wave with a peak value of $8 \mathrm{kV}$ and a frequency of $26 \mathrm{kHz}$ under ambient air. For exsitu $I_{2}$ doping, samples of the deposited both PANI and PTh thin films were placed in a sealed glass container containing $2 \mathrm{~g}$ of solid $\mathrm{I}_{2}$ crystals.

\section{Results and Discussion}

Fig. 1 shows the scanning electron microscopy (SEM) images of the $I_{2}$ doped PANI and PTh thin films. The both PANI and PTh films with doping have nanofibers and nanoparticles morphology with irregularly cross-linked and highly porous networks.

Fig. 2 shows the gas responses of the PANI nanofibers with nanoparticle thin films on the

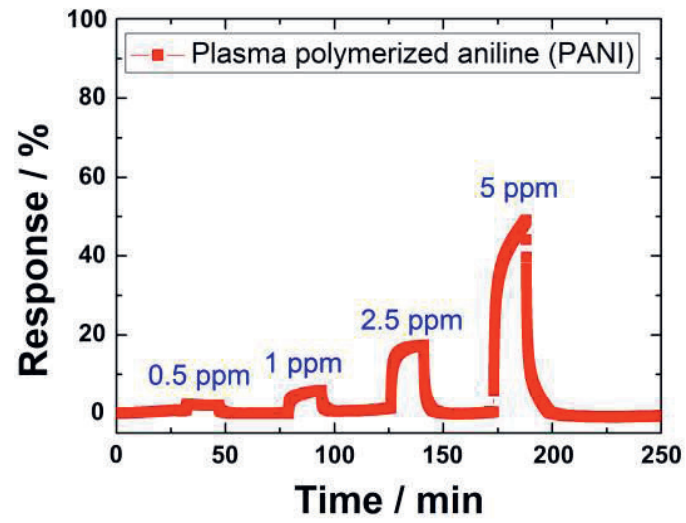

Fig. 2. Response curve of PANI thin films on substrates of interdigitated electrodes prepared using novel APPJs from $0.5 \mathrm{ppm}$ to $5 \mathrm{ppm}$ of $\mathrm{NO}_{2}$ concentration at room temperature.

substrates of interdigitated electrodes prepared using novel APPJs from $0.5 \mathrm{ppm}$ to $5 \mathrm{ppm}$ of the $\mathrm{NO}_{2}$ concentration at room temperature (under $30{ }^{\circ} \mathrm{C}$ ). The new nanostructured conductive porous polymer sensor prepared using novel APPJs polymerization technique showed the excellent $\mathrm{NO}_{2}$-sensing properties at room temperature. The detailed novel APPJs device, sensor resistance, SEM images, TEM images, XPS, GPC, TOF-SIMS, plasma physics, discharge characteristics, and more detailed mechanism are studied and will be discussed in detail.

\section{References}

[1] H. Bai, G. Shi, Gas Sensors Based on Conducting Polymers, Sensors 7, 267-307 (2007); doi: 10.3390/s7030267

[2] D. Nicolas-Debarnot, F. Poncin-Epaillard, Polyaniline as a New Sensitive Layer for Gas Sensors, Analytica Chimica Acta 475, 1-15 (2003); doi: 10.1016/S0003-2670(02)01229-1

[3] S. Virji, J. Huang, R. B. Kaner, B. H. Weiller, Polyaniline Nanofiber Gas Sensors: Examination of Response Mechanisms, Nano Letters 4, 491496 (2004); doi: 10.1021/nl035122e

[4] C.-S. Park, D. H. Kim, B. J. Shin, D. Y. Kim, H.-K. Lee, H.-S. Tae, Conductive Polymer Synthesis with Single-Crystallinity via a Novel Plasma Polymerization Technique for Gas Sensor Applications, Materials 9, 812 (2016); doi: 10.3390/ma9100812

[5] C.-S. Park, E. Y. Jung, D. H. Kim, D. Y. Kim, H.-K. Lee, B. J. Shin, D. H. Lee, H.-S. Tae, Atmospheric Pressure Plasma Polymerization Synthesis and Characterization of Polyaniline Films Doped with and without lodine, Materials 10, 1272 (2017); doi: 10.3390/ma10111272

[6] C.-S. Park, D. Y. Kim, D. H. Kim, H.-K. Lee, B. J. Shin, H.-S. Tae, Humidity-independent Conducting Polyaniline Films Synthesized Using In-situ lodine Doping, Appl. Phys. Lett. 110, 033502 (2017); doi: 10.1063/1.4974222 